\title{
An overview of the knowledge, perception and experience of dentists in Makassar about the utilization of teledentistry as a dental care media
}

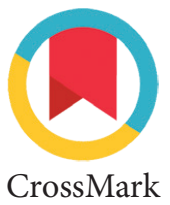

CrossMark

\author{
Rasmidar Samad, 'Burhanuddin D. Pasiga, Nursyamsi, Alex Aryanto
}

Abstract

Objective: To describe the characteristics, knowledge, perceptions, and experiences of dentists regarding the use of teledentistry. Material and Methods: A cross-sectional survey with 87 dentists in Makassar as respondents. The service was carried out by distributing questionnaires in the form of statements and questions regarding the use of teledentistry as a dental care medium. This study used univariate analysis.

Results: Most of the respondents were aged 31-40 years (43.7\%) and $71.3 \%$ of the respondents were women. As many as $60.9 \%$ of respondents have good knowledge about the use of teledentistry. As many as $80.5 \%$ of respondents have a good perception of the use of teledentistry. As many as $58.6 \%$ of respondents have no experience in using teledentistry.

Conclusion: Generally, dentists in Makassar already have a good knowledge in understanding teledentistry as utilization. dental care media. Generally, dentists in Makassar have a good perception in interpreting the use of teledentistry as dental care media. Most of the dentists have no experience in the use of teledentistry as dental care media.
Department of Public Dental Health, Faculty of Dentistry, Hasanuddin University, Makassar, Indonesia
${ }^{*}$ Correspondence to: Rasmidar

Samad, Alex Aryanto, Department of Public Dental Health, Faculty of Dentistry, Hasanuddin University, Makassar, Indonesia

hscrp.samad@gmail.com ; aryantoa18@student.unhas.ac.id

Received: 22 June 2021

Revised: 21 July 20121

Accepted: 30 September 2021

Available Online: 1 December 2021

Keywords: Dentist, Experience, Knowledge, Perception, Teledentistry

Cite this Article: Samad R, Pasiga BD, Nursyamsi, Aryanto A. 2021. An overview of the knowledge, perception, and experience of dentists in Makassar about the utilization of teledentistry as a dental care media. Journal of Dentomaxillofacial Science 6(3): 180-183. D0I: 10.15562/ jdmfs.v6i3.1251

\section{Introduction}

Advances in the use of computers and telecommunications technology are also growing rapidly, so that they can be utilized for digital diagnostic services for analysis and treatment in dentistry. ${ }^{1}$ Health care has changed dramatically in the age of computers, digitization and telecommunications. ${ }^{2}$ The oral health gap is a global problem with most specialist dentists located in urban areas. The limited number of dentists in rural areas means that people do not have access to quality dental care in these areas. Information technology has enabled fast and efficient transmission of information. The development of communication programs has enabled the emergence of a global society and electronic interconnection without geographical boundaries. Just as the use and communication of electronic information technology has evolved over the years, in the field of health, several technological and communication methods have been developed which are called telehealth. The method allows interaction between patients and health care providers over long distances in various areas. ${ }^{3}$

Telemedicine is the use of information-based technology and communication systems to provide health services across geographic distances. ${ }^{4-6}$ This method uses electronic information to support support health services when distance separates health workers from patients. Advances in digital communications, telecommunications and the advent of the internet have made long-distance access to medical services easier. ${ }^{1}$ Although teledentistry is a rapidly growing field, barriers to increasing its use in practice remain. Therefore, the use of teledentistry can have a major impact on improving the oral health status of a region (region). Knowledge, perception, and experience of dental professionals play an important role in the use (utilization) of teledentistry in the present and future. Therefore, the current study was conducted to find out the description of the knowledge, perception, and experience of dentists regarding the use of teledentistry as dental care media. The focus of this study is to explore the knowledge, perceptions, and experiences of practitioners (dentists) in Makassar about the use of teledentistry as a medium for dental and oral health services.

\section{Material and Methods}

The type of research is descriptive observational. The research design used in this study is a crosssectional study. This research was conducted online through an electronic form (google form), so that respondents can fill out forms wherever they are. The population in this study were dentists who 


\section{Table 1 Profile of demographic characteristics of respondents}

\begin{tabular}{llll}
\hline Demography & & $\mathrm{N}$ & $\%$ \\
\hline & $21-30$ & 10 & 11.50 \\
& $31-40$ & 38 & 43.70 \\
Age (Y.O) & $41-50$ & 31 & 35.60 \\
& $51-60$ & 8 & 9.20 \\
Sex & Male-Female & 25 & 28.70 \\
Practice time & & 62 & 71.30 \\
& 5 5 years & 27 & 31.00 \\
& $>10$ years & 27 & 31.00 \\
& & 45 & 51.70 \\
\hline
\end{tabular}

Table 2 Responses distribution from dentists' knowledge regarding the use of teledentistsry as dental care media

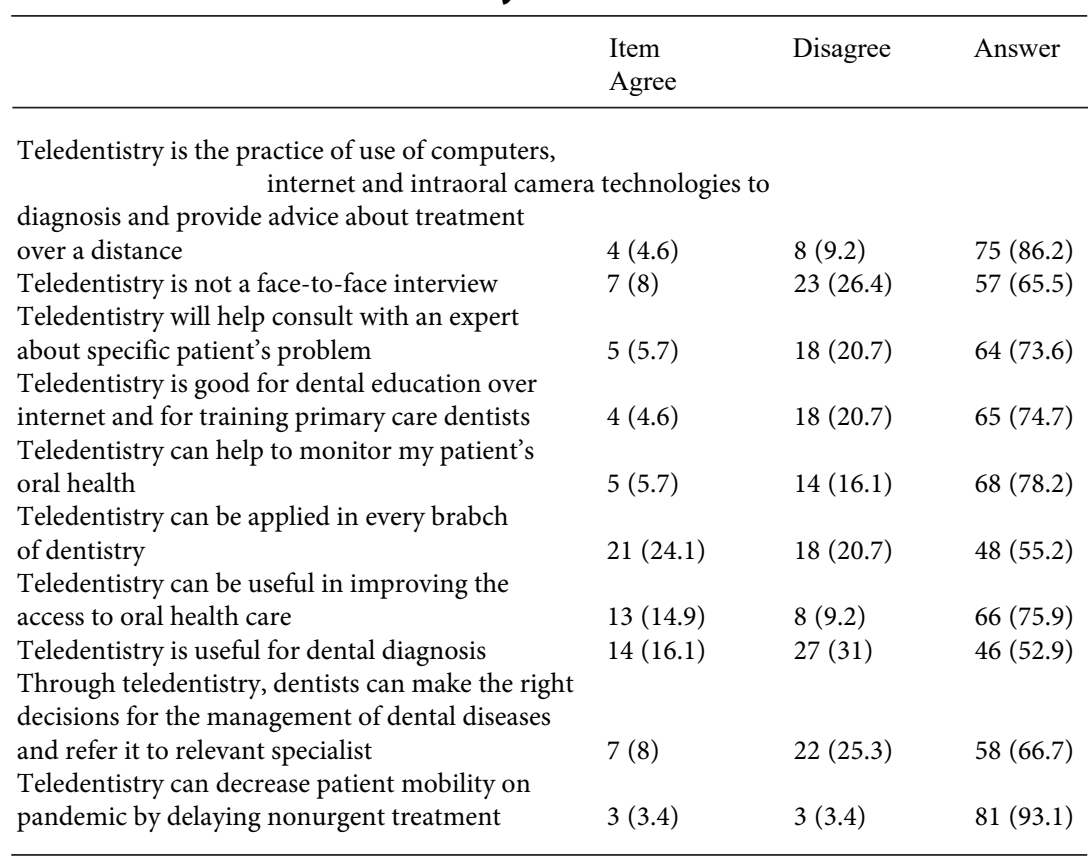

Table 3 Dentist's knowledge about

the use of teledentistsry as dental care media

\begin{tabular}{lll}
\hline Knowledge & $\mathrm{N}$ & $\%$ \\
\hline Less & 34 & 39.1 \\
Good & 53 & 60.9 \\
Total & 87 & 100.0 \\
\hline
\end{tabular}

were registered as members of PDGI Makassar Branch, amounting to 711 people. The sampling method used in this study was simple random sampling. The sampling process is carried out by giving each member of the population the same opportunity to become a member of the sample. By using the Slovin formula, the researcher obtained the number of samples. The process of choosing the number of samples $\mathrm{n}$ from population $\mathrm{N}$ is done randomly such as taking a social gathering to obtain a sample of 87 respondents. Data were collected through a questionnaire (Google form) and distributed to respondents. The data collected is primary data. The data collection instruments in this study included: a questionnaire containing the characteristics of the sample, including gender, age, and length of practice, knowledge and attitude questionnaire, computer with Microsoft Excel and SPSS programs.

\section{Results}

In this study, there were 87 dentists according to the number needed when calculating the sample. In this study, the largest number of respondents were aged 31-40 years, as many as 38 respondents. The number of female respondents was more, namely 62 respondents compared to men, namely as many as 25 respondents. In this study, most of the respondents had practiced for more than 10 years, namely 45 respondents table 1.

Table 2 shows distribution of responses from dentists' knowledge regarding the use of teledentistsry as dental care media. This knowledge appears or exists because it is influenced by many factors, namely age, gender, good internet access. This good knowledge can also be supported by advances in technology and telecommunications, so that the latest dental information and technology can be easily obtained, especially by professionals. Perception is a respondent's interpretation of teledentistry. In this study, the perception of dentists was mostly good, i.e. $80.5 \%$. These perceptions include matters relating to the usefulness and advantages of teledentistry.

In table 3, we highlight the dentist's knowledge about the use of teledentistsry as dental care media. We found that teledentistry is an alternative service that combines the fields of dentistry with technology and telecommunications involving the remote exchange of clinical information and images for dental consultation and treatment planning. Teledentistry has the ability to increase access to dental and oral health, and lower its costs and has the potential to eliminate disparities/ equity in oral health care between rural and urban communities.

Responses distribution from dentists' perception regarding the use of teledentistsry as dental care media is depicted in table 4. Advances in science and technology of dentistry are often a challenge for dentists to keep up with all the developments that occur simultaneously. One of the developments in dentistry is the use of longdistance communication 
Table 4 Responses distribution from dentists' perception regarding the use of teledentistsry as dental care media

\begin{tabular}{lll}
\hline \multicolumn{1}{c}{ Item } & \multicolumn{2}{c}{ Answer } \\
\cline { 2 - 3 } & Yes (\%) & \multirow{2}{*}{ No (\%) } \\
\hline $\begin{array}{l}\text { If you needed a consultation in an oral lesion, } \\
\text { do you think the oral medicine specialist needs }\end{array}$ & & \\
to see the patient? & $70(80.5)$ & $17(9.5)$ \\
Will teledentistry improve dental practice? & $77(88.5)$ & $10(11.5)$ \\
Do you think teledentistry saves time? & $83(95.4)$ & $4(4.6)$ \\
Do you think teledentistry is cost effective? & $66(75.9)$ & $21(24.1)$ \\
\hline
\end{tabular}

Table 5 Perception of dentists in the utilization of teledentistsry as dental care media

\begin{tabular}{lll}
\hline Perception & $\mathrm{N}$ & $\%$ \\
\hline Less & 17 & 19.5 \\
Good & 70 & 80.5 \\
Total & 87 & 100.0 \\
\hline
\end{tabular}

technology which has become a breakthrough in dental and oral health care in the modern era which is now called teledentistry. Advances in communication and information technology not only improve the quality of dental care for patients, but also enable remote care, especially for people who live far from health care centers or dentists. In table 5, we highlight the perception of dentists in the utilization of teledentistsry as dental care media. The foundation of modern teledentistry is the use of the internet and high-speed network connections, which can help teledentistry to become one of the dental sciences needed to enter the new digital era, although teledentistry can also be applied by utilizing simple technology.

\section{Discussion}

Knowledge plays an important role in the use of teledentistry, both by professionals and ordinary people. With knowledge, someone who previously did not or did not know, through the learning process, then that person has knowledge about something. In the study of the previous chapter, it was found that the majority of dentists' knowledge about the use of teledentistry was good, namely $60.9 \%$. Respondents' knowledge of teledentistry is positive (good), as much as $88 \%{ }^{3}$ A similar study was also conducted by Nagarajappa et al. ${ }^{2}$ in Udaipur showing that dentists have good knowledge of teledentistry. ${ }^{2}$

Knowledge is the result of knowing and this occurs after people have sensed a certain object. Knowledge or cognition is a very important domain for the formation of one's mind. Knowledge is a source of information and discovery which is a creative process to get new knowledge. Knowledge is closely related to science. To have one's knowledge, individuals need to carry out a process called learning. ${ }^{7}$ Perception in general is the process of acquiring, interpreting, selecting and arranging sensory information. Perception is the process of seeking information to be understood using the senses. In perception contains a process within ourselves to know and evaluate the extent to which we know other people. In this process the sensitivity in a person to the surrounding environment begins to appear. The perspective will determine the impression that results from the perception process. ${ }^{8}$ Experience is an event that is inherent and related to one another with life. Experience includes things or events experienced by humans in the course of their lives that can be learned and learned by someone. Experience is the result of an analysis of the collection of senses possessed by humans, in other words, experience is an event that is caught by the five senses stored in memory. It can be obtained and felt when new or longstanding events occur, which can be shared with anyone for guidance or learning. ${ }^{9}$

Research conducted by Almazrooa et al. ${ }^{10}$ recorded the perceptions of the respondents. The result obtained by Almazrooa et al..$^{10}$ is that the field of oral radiology is the best specialty that has benefits in teledentistry. It was also found that dentists have a perception that teledentistry can save patients time and costs when performing dental treatment. ${ }^{10} \mathrm{In}$ the study also found that the usefulness teledentistry is to help communication between peers, helps in showing and referring new patients, improve service to patients, improve patient satisfaction, help improve the diagnosis of cases and help in making a better medical record. ${ }^{10}$

Teledentistry can also be used for education and counseling to increase public awareness of dental and oral health. This concept has evolved over the last few years and is becoming an important component of healthcare delivery worldwide. ${ }^{10}$ Teledentistry, similar to telemedicine, has emerged as a new tool with promising benefits for various dental disciplines including endodontics, orthodontics, oral surgery, and pediatric dentistry. This has the potential to improve access to and delivery of oral health care in rural and disadvantaged areas. In addition, teledentistry has the potential to save resources and reduce overall healthcare costs. An 
Australian study reported potential savings of up to 40 million dollars per year if teledentistry was applied to screening children at low caries risk. In diagnostic dentistry, proper diagnosis of oral lesions, including oral cancer, can be challenging, especially in underserved communities with limited access to specialized dental care. Therefore, teledentistry can fill this gap and raise the standard of care. ${ }^{10}$

Teledentistry facilitates dental care, instruction, and remote education using technology without direct face-to-face contact with patients. ${ }^{9}$ Teledentistry is the use of communication technology (in the form of electronic medical records, videos, digital photos, smartphones/tablets/ laptops/computers supported by webcams) as supporting media in sending treatment plans, diagnoses, consultations, as well as providing information and education from doctors to patients thus becoming a relationship between patients and dental health service providers, such as doctors and nurses. ${ }^{11,12}$ The foundation of modern teledentistry is the use of the internet and high-speed network connections, which can help teledentistry to become one of the dental sciences needed to enter the new digital era. ${ }^{1}$ The Internet is the foundation of a system teledentistry modern, it is up-to-date, fast and can carry large amounts of data. There are many reasons "why internet-based teledentistry takes precedence over other means of communication." The reason is that information is fast, low-cost, efficient, recorded consultation, at least face-to-face, and can communicate with several participants at once. While the potential drawbacks are the need for proper training, pressure to respond quickly and misunderstandings in receiving information.

\section{Conclusion}

In general, dentists in Makassar have good knowledge in understanding the use of teledentistry as dental care. Dentists in Makassar have a good perception in interpreting the use of teledentistry as dental care. Most dentists in Makassar have no experience in using teledentistry as dental care. Dentists in Makassar City should not hesitate to use teledentistry as an alternative in providing dental and oral health services. Dentists in Makassar City can take and seek a lot of experience in using teledentistry as a dental care medium. Dentists in Makassar should not only use teledentistry in certain sector of dentistry, but in all branches of dentistry.

\section{Acknowledgment}

The authors thank all the participants, especially dentists who are registered in PDGI Makassar branch, Indonesia, for the support and facilitation in this study.

\section{Conflict of Interest}

The authors report no conflict of interest.

\section{References}

1. Astoeti TE, Widyarman AS. Teledentistry Faculty of Dentistry Trisakti University; Jakarta: 2020. p. 1-30.

2. Nagarajappa R, Aapaliya P, Sharda AJ, et al. Teledentistry: Knowledge and attitudes among dentists in Udaipur, India. Oral Health Dent Manag 2013;12: 502.

3. Murererehe J, Uwambaye P, Isyagi M, et al. Knowledge, attitude and practices of dental professionals in Rwanda towards the benefits and application of teledentistry. F Med Health Science 2017;4: 39.

4. Lankarani KB, Ghahramani S, Zakeri M, et al. Lessons learned from national health accounts in Iran: highlighted evidence for policymakers. Shiraz E-Med J 2015;16: e27868.

5. Daniel SJ, Kumar S. Teledentistry: A key component in access to care. J Evid Based Dent Prac 2014;14: 201-208.

6. Estai M, Kruger E, Tennant M. Optimizing patient referrals to dental consultants: Implication of teledentistry in rural settings. Aust Med J 2016;9: 249.

7. Notoatmodjo, Soekidjo. Health Education and Behavior. Jakarta: Rineka Cipta. 2003

8. Listyana R, Hartono Y. People's perceptions and attitudes towards the Javanese calendar in determining the time of marriage (case study in Jonggrang Village, West District, Magetan Regency in 2013). Agastya J 2015;5: 121-122.

9. Prasetya A, Hidayat D. Experiences of informal workers in the Midst of the covid-19 pandemic in Bandung City. J Komaskam 2020;2: 20-21.

10. Almazrooa SA, Mansour GA, Alhamed SA, et al. The application of teledentistry for Saudi patients' care: A national survey study. J Dent Sci 2021;16: 280-286.

11. Amar B. Teledentistry: A novel tool in our arsenal to combat COVID-19. Int J Health Care Qual Assur 2020;4: 77-80.

12. Gebreegzabher S. Teledentistry. J Res Prog 2019;2: 84-89. 